\title{
Convergence rates in homogenization of $p$-Laplace equations
}

Jie Zhao ${ }^{1 *}$ and Juan Wang ${ }^{1}$

${ }^{*}$ Correspondence:

kaifengajie@163.com

${ }^{1}$ College of Science, Zhongyuan

University of Technology,

Zhengzhou, China

\begin{abstract}
This paper is concerned with homogenization of $p$-Laplace equations with rapidly oscillating periodic coefficients. The main difficulty of this work is due to the nonlinear structure in this field concerning $p$-Laplace equations itself. Utilizing the layer and co-layer type estimates as well as homogenization techniques, we establish the desired error estimates. As a consequence, we obtain the rates of convergence for solutions in $W_{0}^{1, P}$ as well as $L^{P}$. Meanwhile, our convergence rate results do not involve the higher derivatives any more. This may be viewed as rather surprising. The novelty of this work is that it seems to find a new analysis method in quantitative homogenization.
\end{abstract}

MSC: Primary 35B27; secondary $35 J 15$

Keywords: Homogenization; Convergence rates; $p$-Laplace equations; Oscillating

\section{Introduction}

In this paper, we shall establish the rates of convergence for $p$-Laplace equations with rapidly oscillating periodic coefficients. More precisely, let $\Omega$ be a bounded Lipschitz domain in $\mathbb{R}^{n}$. Suppose that $u_{\varepsilon} \in W^{1, p}(\Omega)$, for any $1 \leq p<\infty$, is a weak solution to the following problem:

$$
\begin{cases}L_{\varepsilon} u_{\varepsilon}=-\operatorname{div}\left(A(x / \varepsilon)\left|\nabla u_{\varepsilon}\right|^{p-2} \nabla u_{\varepsilon}\right)=F & \text { in } \Omega, \\ u_{\varepsilon}=f & \text { on } \partial \Omega .\end{cases}
$$

Throughout this paper, the summation convention is used. We assume that the matrix $A(y)=\left(a_{i j}(y)\right)$ with $1 \leq i, j \leq n$, is real, bounded measurable, and satisfies the following conditions.

- Periodicity conditions: for any $y \in \mathbb{R}^{n}$ and $Y=[0,1)^{n} \simeq \mathbb{R}^{n} / \mathbb{Z}^{n}$,

$$
A(y+Y)=A(y)
$$

- Coerciveness and growth conditions: there exists a $\lambda>0$, for any $y \in \mathbb{R}^{n}$ and $\xi, \xi^{\prime} \in \mathbb{R}^{n}$,

$$
\begin{aligned}
\lambda\left(|\xi|+\left|\xi^{\prime}\right|\right)^{p-2}\left|\xi-\xi^{\prime}\right|^{2} & \leq\left\langle A(y)|\xi|^{p-2} \xi-A(y)\left|\xi^{\prime}\right|^{p-2} \xi^{\prime}, \xi-\xi^{\prime}\right\rangle \\
& \leq \frac{1}{\lambda}\left(|\xi|+\left|\xi^{\prime}\right|\right)^{p-2}\left|\xi-\xi^{\prime}\right|^{2}
\end{aligned}
$$

(c) The Author(s) 2019. This article is distributed under the terms of the Creative Commons Attribution 4.0 International License (http://creativecommons.org/licenses/by/4.0/), which permits unrestricted use, distribution, and reproduction in any medium, provided you give appropriate credit to the original author(s) and the source, provide a link to the Creative Commons license, and indicate if changes were made. 
- Smoothness conditions: with $1 / p+1 / p^{\prime}=1$,

$$
F \in W^{-1, p^{\prime}}(\Omega), \quad f \in W^{1, p}(\partial \Omega) .
$$

It is well known that the solution $u_{\varepsilon} \rightarrow u_{0}$ weakly in $W^{1, p}(\Omega)$, as $\varepsilon \rightarrow 0$, where $u_{0}$ is the solution to the homogenized problem

$$
\begin{cases}L_{0} u_{0}=-\operatorname{div}\left(Q\left|\nabla u_{0}\right|^{p-2} \nabla u_{0}\right)=F & \text { in } \Omega, \\ u_{0}=f & \text { on } \partial \Omega .\end{cases}
$$

The $Q$ is a constant matrix, defined by

$$
Q=\int_{Y}\left[A(y)|\nabla \chi(y)+1|^{p-2}(\nabla \chi(y)+1)\right] d y
$$

where the corrector $\chi(y)$ satisfies the following cell problem:

$$
\left\{\begin{array}{l}
\operatorname{div}\left[A(y)|\nabla \chi(y)+1|^{p-2}(\nabla \chi+1)\right]=0 \quad \text { in } Y, \\
\int_{Y} \chi(y) d y=0 .
\end{array}\right.
$$

Recently, there has been published much classical work about convergence of solutions for linear operators in homogenization with the various settings. In 2011, Gérard and Masmoudi [4] obtained the $L^{2}$ convergence rate for the boundary layers Neumann problems. In 2012, Kenig, Lin and Shen [7] obtained $L^{2}$ as well as $H^{\frac{1}{2}}$ convergence rates for the elliptic oscillating operators. In 2013, Aleksanyan, Shahgholian and Sjölin [1,2] proved pointwise as well as $L^{p}$ estimates for fixed operators and oscillating Dirichlet boundary data. In 2014, Kenig, Lin and Shen [8] established $W^{k, p}$ convergence rates, via the asymptotic behavior of the Green or Neumann functions. In 2015, the first author [24] obtained the pointwise as well as $W^{1, p}$ convergence rates for fixed operators and oscillating Neumann boundary data. In 2015, Gu [5] also proved convergence rates in $L^{2}$ and $H^{1}$ for linear Stokes systems. In 2016, Shen [18] proved the $L^{2}$ convergence rate for the mixed Dirichlet-Neumann boundary value problems. In 2018, Niu and Xu [11] got the $L^{2}$ convergence rate for $2 m$ thorder equations with periodic oscillating coefficients.

The nonlinear operators case in homogenization have also been studied extensively. Piat and Deferanceschi [15] have obtained convergence weakly in $W^{1, p}$ for the quasi-linear monotone operator. Pastukhova [14] considered nonlinear equations of monotone type with multiscale coefficients, and established the $L^{2}$ convergence rate. Recently, Wang, Xu and Zhao [21] studied the quasilinear elliptic equations and obtained an error estimate in $L^{2}$. We refer the reader to see $[3,6,10,16,23]$ and their references for more results about nonlinear problems in homogenization.

The motivation for studying this paper is inspired by the problems raised by Wang, $\mathrm{Xu}$ and Zhao in [22] for the $p$-Laplace type equations. The aim of the paper is to obtain the accurate convergence rates of solutions for the classical $p$-Laplace equations with rapidly oscillating periodic coefficients. Thanks to the layer and co-layer type estimates, we could handle the different ingredients in the integral by energy methods. The similar procedures could be found in [9] or [12], which were used to analyze the spatial and mechanical properties for solutions of reflecting the microstructure of the materials. 
The following are the main results of this paper.

Theorem 1 Let $\Omega$ be a bounded Lipschitz domain in $\mathbb{R}^{n}$. Suppose that $u_{\varepsilon} \in W^{1, p}(\Omega)$ and $u_{0} \in W^{1, q}(\Omega)$, with $q>p \geq 4$, are the weak solutions of the problems (1.1) and (1.5), respectively. Then, under the assumptions (1.2)-(1.4), there exists a constant $C$ such that

$$
\left\|u_{\varepsilon}-u_{0}-\varepsilon \chi T_{\varepsilon}\left(\eta_{\varepsilon} \nabla u_{0}\right)\right\|_{W_{0}^{1, p}(\Omega)} \leq C \varepsilon^{\frac{1}{p}-\frac{1}{q}}\left\|\nabla u_{0}\right\|_{L^{q}(\Omega)},
$$

where $T_{\varepsilon}$ is the smoothing operator, and $\eta_{\varepsilon}$ is a cut-off function.

Theorem 2 Under the same conditions as Theorem 1, then there exists a constant $C$, with some $q>p \geq 4$, such that

$$
\left\|u_{\varepsilon}-u_{0}\right\|_{L^{p}(\Omega)} \leq C \varepsilon^{\frac{1}{p}-\frac{1}{q}}\left\|\nabla u_{0}\right\|_{L^{q}(\Omega)} .
$$

The astute reader may have already noticed that our convergence rate result in Theorem 1 , which do not involve the higher derivatives any more. This may be viewed as rather surprising, even though in the linear case. The novelty of this work is that it seems to find a new analysis method, which depends on the layer and co-layer type estimates, in quantitative homogenization. To the best of our knowledge, there are few contributions in the field concerning $p$-Laplace equations in homogenization.

The rest of the paper is organized as follows. Section 2 contains some basic notations and useful propositions which will play crucial roles to obtain convergence rates. In Sect. 3, we show that the solution $u_{\varepsilon}$ of $p$-Laplace equations is convergent to the solution $u_{0}$ of the corresponding homogenized problems, this is based on the energy method as well as using homogenization tools.

\section{Preliminaries}

We begin by specifying our notations.

Let $B_{r}(x)$ denote an open ball with center $x$ and radius $r . \Omega_{\varepsilon}=\{x \in \Omega$ : $\operatorname{dist}(x, \partial \Omega)>\varepsilon\}$, we also call it the co-layer part of $\Omega$, associated with so-called layer part is denoted by $\Omega \backslash \Omega_{\varepsilon}$. Set $\eta_{\varepsilon} \in C_{0}^{\infty}(\Omega)$ is a cut-off function, satisfying $\eta_{\varepsilon}=1$ in $\Omega_{\varepsilon}, \eta_{\varepsilon}=0$ outside $\Omega_{\varepsilon}$ and $\left|\nabla \eta_{\varepsilon}\right| \leq C / \varepsilon$. In the whole paper, we use $C$ to denote positive constant which may vary in different formulas.

Proposition 2.1 Let $F=\left(F_{1}, F_{2}, \ldots, F_{n}\right) \in L^{p}(Y)$. Suppose that $\int_{Y} F_{j}(y) d y=0$ and $\operatorname{div} F(y)=$ 0 in $Y$. Then there exists $\Phi_{i j} \in W^{1, p}(Y)$ such that $\Phi_{i j}=-\Phi_{j i}$ and $F_{j}=\frac{\partial \Phi_{i j}}{\partial y_{i}}$.

This proposition is well known. It is called the technique of flux correctors. The linear operator case is well known (see for example [7], Lemma 3.1). Let $f_{j} \in W^{2, p}(Y)$ be the solution to the cell problem $\triangle f_{j}=F_{j}$ in Y. Then we could define $\Phi_{i j}(y)=\frac{\partial}{\partial y_{i}}\left[f_{j}(y)\right]-\frac{\partial}{\partial y_{j}}\left[f_{i}(y)\right]$. From an energy estimate, we may get the desired properties.

Recently, the smoothing operator was introduced by Suslina in $[19,20]$. Meanwhile, applying the smoothing operator to get error estimates was first established by Shen in [17]. Next, we will introduce the smoothing operator and its properties. This work is to extend the usage of smoothing operator to the case of $p$-Laplace equations, of independent interest itself. 
Definition 2.2 Fix $\psi \in C_{0}^{\infty}\left(B_{1}(0)\right)$ such that $\psi \geq 0$ and $\int_{\mathbb{R}^{n}} \psi d x=1$. Define operator $T_{\varepsilon}$ on $L^{2}$ as

$$
T_{\varepsilon}(u)(x)=u * \psi_{\varepsilon}=\int_{\mathbb{R}^{n}} u(x-y) \psi_{\varepsilon}(y) d y,
$$

where $\psi_{\varepsilon}(x)=\varepsilon^{-n} \psi(x / \varepsilon)$. We call it the smoothing operator.

Proposition 2.3 Let $u_{0} \in W^{1, p}(\Omega)$ and a periodic function $f \in L^{p}(Y)$, for some $1<p<\infty$. Then we have

$$
\left\|f(\cdot / \varepsilon) T_{\varepsilon}\left(u_{0}\right)\right\|_{L^{p}(\Omega)} \leq C\|f\|_{L^{p}(Y)}\left\|u_{0}\right\|_{L^{p}(\Omega)}
$$

and

$$
\left\|u_{0}-T_{\varepsilon}\left(\nabla u_{0}\right)\right\|_{L^{p}\left(\Omega_{\varepsilon}\right)} \leq C \varepsilon\left\|\nabla u_{0}\right\|_{L^{p}\left(\Omega_{\varepsilon}\right)}
$$

These estimates could be proved by Fubini's theorem and Hölder's inequality. We refer the reader to $[13,17]$ or $[18]$ for the detailed proof.

The main interest of the present work is to attempt to find a new approach to analyzing the error estimates for homogenization problems. Fortunately, it may be a new way to derive rates of convergence, via the co-layer and layer type estimates.

Proposition 2.4 (Co-layer and layer type estimates) If $u_{0} \in W^{1, p}(\Omega)$ for some $q>p>1$, then we have estimates

$$
\begin{aligned}
& \int_{\Omega \backslash \Omega_{\varepsilon}}\left|\nabla u_{0}\right|^{p} d x \leq C \varepsilon^{1-\frac{p}{q}}\left(\int_{\Omega}\left|\nabla u_{0}\right|^{q} d x\right)^{\frac{p}{q}}, \\
& \int_{\Omega_{\varepsilon}}\left|\nabla^{2} u_{0}\right|^{2}\left|\cdot \nabla u_{0}\right|^{p-2} d x \leq C \varepsilon^{-1-\frac{p}{q}}\left(\int_{\Omega}\left|\nabla u_{0}\right|^{q} d x\right)^{\frac{p}{q}},
\end{aligned}
$$

and

$$
\int_{\Omega_{\varepsilon}}\left|\nabla^{2} u_{0}\right|^{p} d x \leq C \varepsilon^{1-\frac{p}{q}-p}\left(\int_{\Omega}\left|\nabla u_{0}\right|^{q} d x\right)^{\frac{p}{q}} .
$$

These estimates will play crucial roles to obtain convergence rates in the present paper, and they also do not involve the higher derivatives any more. These estimates could be derived by regularity estimates, and they may be found in [22].

\section{Proofs of theorems}

The goal of this section is to establish $W_{0}^{1, p}$ and $L^{p}$ convergence rates of solutions for the $p$-Laplace equations in homogenization.

Set the first-order approximation term

$$
v_{\varepsilon}=u_{0}+\varepsilon \chi T_{\varepsilon}\left(\eta_{\varepsilon} \nabla u_{0}\right) .
$$


We find that

$$
\nabla v_{\varepsilon}=\nabla u_{0}+\nabla \chi T_{\varepsilon}\left(\eta_{\varepsilon} \nabla u_{0}\right)+\varepsilon \chi T_{\varepsilon}\left(\eta_{\varepsilon} \nabla^{2} u_{0}\right)+\varepsilon \chi T_{\varepsilon}\left(\nabla \eta_{\varepsilon} \nabla u_{0}\right)
$$

In view of the fact that, for any $\varphi \in C_{0}^{\infty}(\Omega)$,

$$
\int_{\Omega} A(x / \varepsilon)\left|\nabla u_{\varepsilon}\right|^{p-2} \nabla u_{\varepsilon} \cdot \nabla \varphi d x=\int_{\Omega} Q\left|\nabla u_{0}\right|^{p-2} \nabla u_{0} \cdot \nabla \varphi d x
$$

we obtain

$$
\begin{aligned}
\int_{\Omega}[ & \left.A(x / \varepsilon)\left|\nabla u_{\varepsilon}\right|^{p-2} \nabla u_{\varepsilon}-A(x / \varepsilon)\left|\nabla v_{\varepsilon}\right|^{p-2} \nabla v_{\varepsilon}\right] \cdot \nabla \varphi d x \\
= & \int_{\Omega}\left[Q\left|\nabla u_{0}\right|^{p-2} \nabla u_{0}-Q\left|T_{\varepsilon}\left(\eta_{\varepsilon} \nabla u_{0}\right)\right|^{p-2} T_{\varepsilon}\left(\eta_{\varepsilon} \nabla u_{0}\right)\right] \cdot \nabla \varphi d x \\
& +\int_{\Omega}\left[Q\left|T_{\varepsilon}\left(\eta_{\varepsilon} \nabla u_{0}\right)\right|^{p-2} T_{\varepsilon}\left(\eta_{\varepsilon} \nabla u_{0}\right)-A(x / \varepsilon)\left|T_{\varepsilon}\left(\eta_{\varepsilon} \nabla u_{0}\right)\right|^{p-2}\right. \\
& \left.\cdot T_{\varepsilon}\left(\eta_{\varepsilon} \nabla u_{0}\right)|\nabla \chi+1|^{p-2}(\nabla \chi+1)\right] \cdot \nabla \varphi d x \\
& +\int_{\Omega}\left[A(x / \varepsilon)\left|T_{\varepsilon}\left(\eta_{\varepsilon} \nabla u_{0}\right)\right|^{p-2} T_{\varepsilon}\left(\eta_{\varepsilon} \nabla u_{0}\right)|\nabla \chi+1|^{p-2}(\nabla \chi+1)\right. \\
& \left.-A(x / \varepsilon)\left|\nabla v_{\varepsilon}\right|^{p-2} \nabla v_{\varepsilon}\right] \cdot \nabla \varphi d x \\
\doteq & I_{1}+I_{2}+I_{3} .
\end{aligned}
$$

To estimate $I_{1}$, we note Proposition 2.3, and Proposition 2.4 for the co-layer and layer type estimates, showing that

$$
\begin{aligned}
\left|I_{1}\right| & \leq C \int_{\Omega}\left|\nabla u_{0}-T_{\varepsilon}\left(\eta_{\varepsilon} \nabla u_{0}\right)\right|\left(\left|\nabla u_{0}\right|+\left|T_{\varepsilon}\left(\eta_{\varepsilon} \nabla u_{0}\right)\right|\right)^{p-2} \cdot|\nabla \varphi| d x \\
& \leq C \int_{\Omega \backslash \Omega_{\varepsilon}}\left|\nabla u_{0}\right|^{p-1}|\nabla \varphi| d x+C \int_{\Omega_{\varepsilon}}\left|\nabla u_{0}-T_{\varepsilon}\left(\eta_{\varepsilon} \nabla u_{0}\right)\right|\left|\nabla u_{0}\right|^{p-2}|\nabla \varphi| d x \\
& \leq C\left(\int_{\Omega \backslash \Omega_{\varepsilon}}\left|\nabla u_{0}\right|^{p} d x\right)^{1-\frac{1}{p}}+C \int_{\Omega_{\varepsilon}}\left|\eta_{\varepsilon} \nabla u_{0}-T_{\varepsilon}\left(\eta_{\varepsilon} \nabla u_{0}\right)\right|\left|\nabla u_{0}\right|^{p-2}|\nabla \varphi| d x \\
& \leq C\left(\int_{\Omega \backslash \Omega_{\varepsilon}}\left|\nabla u_{0}\right|^{p} d x\right)^{1-\frac{1}{p}}+C\left(\int_{\Omega_{\varepsilon}}\left|\eta_{\varepsilon} \nabla u_{0}-T_{\varepsilon}\left(\eta_{\varepsilon} \nabla u_{0}\right)\right|^{\frac{p}{p-1}}\left|\nabla u_{0}\right|^{\frac{p(p-2)}{p-1}} d x\right)^{1-\frac{1}{p}} \\
& \leq C\left(\int_{\Omega \backslash \Omega_{\varepsilon}}\left|\nabla u_{0}\right|^{p} d x\right)^{1-\frac{1}{p}}+C \varepsilon\left(\int_{\Omega_{\varepsilon}}\left|\nabla^{2} u_{0}\right|^{\frac{p}{p-1}}\left|\nabla u_{0}\right|^{\frac{p(p-2)}{p-1}} d x\right)^{1-1 / p} \\
& \leq C\left(\int_{\Omega \backslash \Omega_{\varepsilon}}\left|\nabla u_{0}\right|^{p} d x\right)^{1-\frac{1}{p}}+C \varepsilon\left(\int_{\Omega_{\varepsilon}}\left|\nabla^{2} u_{0}\right|^{2}\left|\nabla u_{0}\right|^{p-2} d x\right)^{\frac{1}{2}}\left(\int_{\Omega}\left|\nabla u_{0}\right|^{p} d x\right)^{\frac{p-2}{2 p}} \\
& \leq C \varepsilon^{\left(1-\frac{1}{p}\right)\left(1-\frac{p}{q}\right)}\left(\int_{\Omega}\left|\nabla u_{0}\right|^{q} d x\right)^{\frac{p-1}{q}}+C \varepsilon^{\frac{1}{2}-\frac{p}{2 q}}\left(\int_{\Omega}\left|\nabla u_{0}\right|^{q} d x\right)^{\frac{p}{2 q}}\left(\int_{\Omega}\left|\nabla u_{0}\right|^{q} d x\right)^{\frac{p-2}{2 q}} \\
& \leq C \varepsilon^{\frac{1}{2}\left(1-\frac{p}{q}\right)}\left(\int_{\Omega}\left|\nabla u_{0}\right|^{q} d x\right)^{\frac{p-1}{q}}
\end{aligned}
$$

for some $q>p \geq 2$, where we have used the Hölder inequality. 
Next, we shall estimate $I_{2}$. Let

$$
F(y, \xi)=Q\left|T_{\varepsilon}(\xi)\right|^{p-2} T_{\varepsilon}(\xi)-A(y)\left|T_{\varepsilon}(\xi)\right|^{p-2} T_{\varepsilon}(\xi)|\chi(y)+1|^{p-2}(\nabla \chi(y)+1)
$$

Note that $F(\cdot, \xi)$ is a periodic function with the first variable and satisfies the conditions of Proposition 2.1. Then there exists $\Phi(\cdot, \xi)$, such that $\Phi_{i j}=-\Phi_{j i}$, and

$$
Q\left|T_{\varepsilon}(\xi)\right|^{p-2} T_{\varepsilon}(\xi)-A(y)\left|T_{\varepsilon}(\xi)\right|^{p-2} T_{\varepsilon}(\xi)|\chi(y)+1|^{p-2}(\nabla \chi(y)+1)=\operatorname{div}_{y} \Phi(y, \xi) .
$$

Thus, it gives

$$
\begin{aligned}
I_{2}= & \int_{\Omega} F(x, x / \varepsilon) \cdot \nabla \varphi d x \\
= & \int_{\Omega} \operatorname{div}_{y} \Phi(y, \xi) \cdot \nabla \varphi d x \\
= & \int_{\Omega} \frac{\partial}{\partial y_{i}}\left(\Phi_{i j}(y, \xi)\right) \cdot \frac{\partial \varphi}{\partial x_{j}} d x \\
= & -\int_{\Omega} \frac{\partial}{\partial x_{i}}\left(\varepsilon \Phi_{i j}(x, x / \varepsilon)\right) \cdot \frac{\partial \varphi}{\partial x_{j}} d x+\int_{\Omega} \frac{\partial}{\partial y_{i}}\left(\Phi_{i j}(y, \xi)\right) \cdot \frac{\partial \varphi}{\partial x_{j}} d x \\
& +\int_{\Omega} \varepsilon \Phi_{i j}(x, x / \varepsilon) \cdot \frac{\partial \varphi}{\partial x_{i} \partial x_{j}} d x \\
= & \int_{\Omega}\left[\frac{\partial}{\partial y_{i}}\left(\Phi_{i j}(y, \xi)\right)-\frac{\partial}{\partial x_{i}}\left(\varepsilon \Phi_{i j}(x, x / \varepsilon)\right)\right] \cdot \frac{\partial \varphi}{\partial x_{j}} d x,
\end{aligned}
$$

where we have used the divergence theorem and the antisymmetry of $\Phi_{i j}$.

As a result, using Proposition 2.4 again, we get

$$
\begin{aligned}
\left|I_{2}\right| & \leq C \int_{\Omega}\left|\nabla_{y} \Phi(y, \xi)-\nabla_{x} \Phi(x, x / \varepsilon)\right| \cdot|\nabla \varphi| d x \\
& \leq C \varepsilon \int_{\Omega_{\varepsilon}}\left|T_{\varepsilon}\left(\nabla^{2} u_{0}\right)\right| \cdot\left|\nabla u_{0}\right|^{p-2} \cdot|\nabla \varphi| d x \\
& \leq C \varepsilon\left(\int_{\Omega}\left|\nabla u_{0}\right|^{p} d x\right)^{1-\frac{2}{p}}\left(\int_{\Omega_{\varepsilon}}\left|T_{\varepsilon}\left(\nabla^{2} u_{0}\right)\right|^{p} d x\right)^{\frac{1}{p}} \\
& \leq C \varepsilon\left(\int_{\Omega}\left|\nabla u_{0}\right|^{q} d x\right)^{\frac{p-2}{q}}\left(\int_{\Omega_{\varepsilon}}\left|\nabla^{2} u_{0}\right|^{p} d x\right)^{\frac{1}{p}} \\
& \leq C \varepsilon^{\frac{1}{p}-\frac{1}{q}}\left(\int_{\Omega}\left|\nabla u_{0}\right|^{q} d x\right)^{\frac{p-1}{q}}
\end{aligned}
$$

for some $q>p$.

For $I_{3}$, it follows that

$\left|I_{3}\right|$

$$
\begin{aligned}
& \leq C \int_{\Omega}\left|(\nabla \chi+1) T_{\varepsilon}\left(\eta_{\varepsilon} \nabla u_{0}\right)-\nabla v_{\varepsilon}\right|\left(\left|(\nabla \chi+1) T_{\varepsilon}\left(\eta_{\varepsilon} \nabla u_{0}\right)\right|+\left|\nabla v_{\varepsilon}\right|\right)^{p-2} \cdot|\nabla \varphi| d x \\
& \leq C \int_{\Omega}\left|\nabla u_{0}-T_{\varepsilon}\left(\eta_{\varepsilon} \nabla u_{0}\right)\right|\left(\left|\nabla u_{0}\right|+\left|(\nabla \chi+1) T_{\varepsilon}\left(\eta_{\varepsilon} \nabla u_{0}\right)\right|\right)^{p-2} \cdot|\nabla \varphi| d x
\end{aligned}
$$




$$
\begin{aligned}
& +C \int_{\Omega}\left|\nabla u_{0}-T_{\varepsilon}\left(\eta_{\varepsilon} \nabla u_{0}\right)\right| \\
& +\left(\left|\nabla \chi T_{\varepsilon}\left(\eta_{\varepsilon} \nabla u_{0}\right)\right|+\left|\varepsilon \chi T_{\varepsilon}\left(\eta_{\varepsilon} \nabla^{2} u_{0}\right)\right|+\left|\varepsilon \chi T_{\varepsilon}\left(\nabla \eta_{\varepsilon} \nabla u_{0}\right)\right|\right)^{p-2}|\nabla \varphi| d x \\
& \quad+C \int_{\Omega}\left|\varepsilon \chi T_{\varepsilon}\left(\eta_{\varepsilon} \nabla^{2} u_{0}\right)+\varepsilon \chi T_{\varepsilon}\left(\nabla \eta_{\varepsilon} \nabla u_{0}\right)\right|\left(\left|(\nabla \chi+1) T_{\varepsilon}\left(\eta_{\varepsilon} \nabla u_{0}\right)\right|+\left|\nabla v_{\varepsilon}\right|\right)^{p-2} \\
& \quad \cdot|\nabla \varphi| d x \\
& \doteq I_{31}+I_{32}+I_{33} .
\end{aligned}
$$

Here, we divide the estimate into three ingredients.

Similar to the estimate of $I_{1}$, we have

$$
\begin{aligned}
\left|I_{31}\right| & \leq C\left(\int_{\Omega \backslash \Omega_{\varepsilon}}\left|\nabla u_{0}\right|^{p} d x\right)^{1-\frac{1}{p}}+C \varepsilon\left(\int_{\Omega_{\varepsilon}}\left|\nabla^{2} u_{0}\right|^{2}\left|\nabla u_{0}\right|^{p-2} d x\right)^{\frac{1}{2}}\left(\int_{\Omega}\left|\nabla u_{0}\right|^{p} d x\right)^{\frac{p-2}{2 p}} \\
& \leq C \varepsilon^{\frac{1}{2}\left(1-\frac{p}{q}\right)}\left(\int_{\Omega}\left|\nabla u_{0}\right|^{q} d x\right)^{\frac{p-1}{q}}
\end{aligned}
$$

Next, we proceed to deal with $I_{32}$ :

$$
\begin{aligned}
\left|I_{32}\right| \leq & C \int_{\Omega}\left|\nabla u_{0}-T_{\varepsilon}\left(\eta_{\varepsilon} \nabla u_{0}\right)\right|\left(\left|T_{\varepsilon}\left(\eta_{\varepsilon} \nabla u_{0}\right)\right|+\left|\varepsilon T_{\varepsilon}\left(\nabla \eta_{\varepsilon} \nabla u_{0}\right)\right|\right)^{p-2}|\nabla \varphi| d x \\
& +C \int_{\Omega}\left|\nabla u_{0}-T_{\varepsilon}\left(\eta_{\varepsilon} \nabla u_{0}\right)\right|\left|\varepsilon \chi T_{\varepsilon}\left(\eta_{\varepsilon} \nabla^{2} u_{0}\right)\right|^{p-2}|\nabla \varphi| d x \\
\leq & C \int_{\Omega \backslash \Omega_{\varepsilon}}\left|\nabla u_{0}\right|^{p-1}|\nabla \varphi| d x+C \int_{\Omega_{\varepsilon}}\left|\nabla u_{0}-T_{\varepsilon}\left(\eta_{\varepsilon} \nabla u_{0}\right)\right|\left|\nabla u_{0}\right|^{p-2}|\nabla \varphi| d x \\
& +C \int_{\Omega_{\varepsilon}}\left|\nabla u_{0}\right| \cdot\left|\varepsilon \chi T_{\varepsilon}\left(\eta_{\varepsilon} \nabla^{2} u_{0}\right)\right|^{p-2}|\nabla \varphi| d x \\
\leq & C \varepsilon^{\frac{1}{2}\left(1-\frac{p}{q}\right)}\left(\int_{\Omega}\left|\nabla u_{0}\right|^{q} d x\right)^{\frac{p-1}{q}}+\left(\int_{\Omega}\left|\nabla u_{0}\right|^{q} d x\right)^{\frac{1}{q}}\left(\int_{\Omega_{\varepsilon}}\left|\varepsilon \chi T_{\varepsilon}\left(\eta_{\varepsilon} \nabla^{2} u_{0}\right)\right|^{p} d x\right)^{1-\frac{2}{p}} \\
\leq & \left.C \varepsilon^{\frac{1}{2}\left(1-\frac{p}{q}\right)}\left(\int_{\Omega}\left|\nabla u_{0}\right|^{q} d x\right)^{\frac{p-1}{q}}+\left.\left(\int_{\Omega}\left|\nabla u_{0}\right|^{q} d x\right)^{\frac{1}{q}} \varepsilon^{\left(1-\frac{p}{q}\right)\left(1-\frac{2}{p}\right)}\left(\int_{\Omega} \mid \nabla u_{0}\right)\right|^{q} d x\right)^{\frac{p-2}{q}} \\
\leq & C \varepsilon^{\frac{1}{2}\left(1-\frac{p}{q}\right)}\left(\int_{\Omega}\left|\nabla u_{0}\right|^{q} d x\right)^{\frac{p-1}{q}},
\end{aligned}
$$

for some $q>p \geq 4$, where we have used Proposition 2.3 and Proposition 2.4.

Last, it remains to handle $I_{33}$ :

$$
\begin{aligned}
\left|I_{33}\right| \leq & C \varepsilon \int_{\Omega_{\varepsilon}}\left|T_{\varepsilon}\left(\nabla^{2} u_{0}\right)+T_{\varepsilon}\left(\nabla u_{0}\right)\right| \cdot\left|\nabla u_{0}\right|^{p-2}|\nabla \varphi| d x \\
& +C \varepsilon^{p-1} \int_{\Omega_{\varepsilon}}\left|T_{\varepsilon}\left(\nabla^{2} u_{0}\right)+T_{\varepsilon}\left(\nabla u_{0}\right)\right|^{p-1}|\nabla \varphi| d x \\
\leq & C \varepsilon\left(\int_{\Omega}\left|\nabla u_{0}\right|^{p} d x\right)^{1-\frac{2}{p}}\left(\int_{\Omega_{\varepsilon}}\left|T_{\varepsilon}\left(\nabla^{2} u_{0}\right)+T_{\varepsilon}\left(\nabla u_{0}\right)\right|^{p} d x\right)^{\frac{1}{p}} \\
& +C \varepsilon^{p-1}\left(\int_{\Omega_{\varepsilon}}\left(\left|T_{\varepsilon}\left(\nabla^{2} u_{0}\right)\right|+\left|T_{\varepsilon}\left(\nabla u_{0}\right)\right|\right)^{p} d x\right)^{1-\frac{1}{p}}
\end{aligned}
$$




$$
\begin{aligned}
\leq & C \varepsilon\left(\int_{\Omega}\left|\nabla u_{0}\right|^{q} d x\right)^{\frac{p-2}{q}}\left[\left(\int_{\Omega}\left|\nabla u_{0}\right|^{q} d x\right)^{\frac{1}{q}}+\left(\int_{\Omega_{\varepsilon}}\left|\nabla^{2} u_{0}\right|^{p} d x\right)^{\frac{1}{p}}\right] \\
& +C \varepsilon^{p-1}\left(\int_{\Omega}\left|\nabla u_{0}\right|^{q} d x\right)^{\frac{p-1}{q}}+C \varepsilon^{p-1}\left(\int_{\Omega_{\varepsilon}}\left|\nabla^{2} u_{0}\right|^{p} d x\right)^{1-\frac{1}{p}} \\
\leq & C\left(\varepsilon+\varepsilon^{\frac{1}{p}-\frac{1}{q}}+\varepsilon^{p-1}+\varepsilon^{(p-1)\left(\frac{1}{p}-\frac{1}{q}\right)}\right)\left(\int_{\Omega}\left|\nabla u_{0}\right|^{q} d x\right)^{\frac{p-1}{q}} \\
\leq & C \varepsilon^{\frac{1}{p}-\frac{1}{q}}\left(\int_{\Omega}\left|\nabla u_{0}\right|^{q} d x\right)^{\frac{p-1}{q}},
\end{aligned}
$$

for some $q>p \geq 4$.

This, together with (3.1)-(3.7), shows that, for some $q>p \geq 4$,

$$
\begin{aligned}
& \left|\int_{\Omega}\left[A(x / \varepsilon)\left|\nabla u_{\varepsilon}\right|^{p-2} \nabla u_{\varepsilon}-A(x / \varepsilon)\left|\nabla v_{\varepsilon}\right|^{p-2} \nabla v_{\varepsilon}\right] \cdot \nabla \varphi d x\right| \\
& \quad \leq C \varepsilon^{\frac{1}{\bar{p}-\frac{1}{q}}}\left(\int_{\Omega}\left|\nabla u_{0}\right|^{q} d x\right)^{\frac{p-1}{q}} .
\end{aligned}
$$

Then let $\varphi=v_{\varepsilon}=u_{\varepsilon}-u_{0}-\varepsilon \chi T_{\varepsilon}\left(\eta_{\varepsilon} \nabla u_{0}\right)$. It gives

$$
\begin{aligned}
& \left\|\nabla\left[u_{\varepsilon}-u_{0}-\varepsilon \chi T_{\varepsilon}\left(\eta_{\varepsilon} \nabla u_{0}\right)\right]\right\|_{L^{p}(\Omega)}^{p-1} \\
& \quad \leq C\left|\int_{\Omega}\left[A(x / \varepsilon)\left|\nabla u_{\varepsilon}\right|^{p-2} \nabla u_{\varepsilon}-A(x / \varepsilon)\left|\nabla v_{\varepsilon}\right|^{p-2} \nabla v_{\varepsilon}\right] \cdot \nabla \varphi d x\right| \\
& \quad \leq C \varepsilon^{\frac{1}{p}-\frac{1}{q}}\left(\int_{\Omega}\left|\nabla u_{0}\right|^{q} d x\right)^{\frac{p-1}{q}} .
\end{aligned}
$$

In view of the fact that $T_{\varepsilon}\left(\eta_{\varepsilon} \nabla u_{0}\right)=0$ in the $\Omega \backslash \Omega_{\varepsilon}$ and the Poincare inequality, this completes the proof of Theorem 1 .

It follows from Theorem 1 and Proposition 2.3, together with Minkowski's inequality, that

$$
\begin{aligned}
\left\|u_{\varepsilon}-u_{0}\right\|_{L^{p}(\Omega)} & \leq C \varepsilon\left\|\chi T_{\varepsilon}\left(\eta_{\varepsilon} \nabla u_{0}\right)\right\|_{L^{p}(\Omega)}+C \varepsilon^{\frac{1}{p}-\frac{1}{q}}\left\|\nabla u_{0}\right\|_{L^{q}(\Omega)} \\
& \leq C \varepsilon\left\|\nabla u_{0}\right\|_{L^{p}(\Omega)}+C \varepsilon^{\frac{1}{p}-\frac{1}{q}}\left\|\nabla u_{0}\right\|_{L^{q}(\Omega)} \\
& \leq C \varepsilon^{\frac{1}{p}-\frac{1}{q}}\left\|\nabla u_{0}\right\|_{L^{q}(\Omega)}
\end{aligned}
$$

with $q>p \geq 4$.

This completes the proof of Theorem 2 .

\section{Acknowledgements}

The author would like to thank the reviewers for their valuable comments and helpful suggestions to improve the quality of this paper. The part of this work was done while the author was visiting school of mathematics and applied statistics, University of Wollongong, Australia.

Funding

This work has been supported by the Natural Science Foundation of China (No. 11626239), the China Scholarship Council (No. 201708410483), as well as the Foundation of Education Department of Henan Province (No. 18A110037). 
Availability of data and materials

Not applicable.

\section{Competing interests}

The authors declare that they have no competing interests.

\section{Authors' contributions}

All authors read and approved the submitted manuscript.

\section{Publisher's Note}

Springer Nature remains neutral with regard to jurisdictional claims in published maps and institutional affiliations.

Received: 29 May 2019 Accepted: 21 August 2019 Published online: 29 August 2019

\section{References}

1. Aleksanyan, H., Shahgholian, H., Sjölin, P.: Applications of Fourier analysis in homogenization of Dirichlet problem I. Pointwise estimates. J. Differ. Equ. 254, 2626-2637 (2013)

2. Aleksanyan, H., Shahgholian, H., Sjölin, P.: Applications of Fourier analysis in homogenization of the Dirichlet problem: $L^{p}$ estimates. Arch. Ration. Mech. Anal. 215, 65-87 (2015)

3. Fusco, N., Moscariello, G.: On the homogenization of quasilinear divergence structure operators. Ann. Mat. Pura Appl. 146, 1-13 (1987)

4. Gérard, D., Masmoudi, N.: Homogenization and boundary layers. Acta Math. 209, 133-178 (2012)

5. Gu, S.: Convergence rates in homogenization of Stokes systems. J. Differ. Equ. 260, 5796-5815 (2016)

6. Jikov, V., Kozlov, S., Oleinik, O.: Homogenization of Differential Operators and Integral Functionals. Springer, Berlin (1994)

7. Kenig, C.E., Lin, F.H., Shen, Z.W.: Convergence rates in $L^{2}$ for elliptic homogenization problems. Arch. Ration. Mech. Anal. 203, 1009-1036 (2012)

8. Kenig, C.E., Lin, F.H., Shen, Z.W.: Periodic homogenization of Green and Neumann functions. Commun. Pure Appl. Math. 67, 1219-1262 (2012)

9. Marin, M., Craciun, E.M., Pop, N.: Considerations on mixed initial-boundary value problems for micropolar porous bodies. Dyn. Syst. Appl. 25, 175-196 (2016)

10. Maso, G.D., Defranceschi, A.: Correctors for the homogenization of monotone operators. Differ. Integral Equ. 3, $1151-1166$ (1990)

11. Niu, W., Xu, Y.: Convergence rates in homogenization of higher order parabolic systems. Discrete Contin. Dyn. Syst. 38, 4203-4229 (2018)

12. Othman, M., Marin, M.: Effect of thermal loading due to laser pulse on thermoelastic porous medium under G-N theory. Results Phys. 7, 3863-3872 (2017)

13. Pakhnin, M.A., Suslina, T.A.: Operator error estimates for the homogenization of the elliptic Dirichlet problem in a bounded domain. St. Petersburg Math. J. 24, 949-976 (2013)

14. Pastukhova, S.: Operator estimates in nonlinear problems of reiterated homogenization. Proc. Steklov Inst. Math. 261, 214-228 (2008)

15. Piat, V.C., Deferanceschi, A.: Homogenization of monotone operators. Nonlinear Anal. 14, 717-732 (1990)

16. Piat, V.C., Maso, G.D., Defranceschi, A.: G-convergence of monotone operators. Ann. Inst. Henri Poincaré, Anal. Non Linéaire 3, 123-160 (1990)

17. Shen, Z.W.: Boundary estimates in elliptic homogenization. Mathematics 10, 653-694 (2017)

18. Shen, Z.W., Zhuge, J.: Convergence rates in periodic homogenization of systems of elasticity. Proc. Am. Math. Soc. $145,1187-1202(2016)$

19. Suslina, T.: Homogenization of the Dirichlet problem for elliptic systems: $L^{2}$-operator error estimates. Mathematika 59 463-476 (2013)

20. Suslina, T.: Homogenization of the Neumann problem for elliptic systems with periodic coefficients. SIAM J. Math Anal. 45, 3453-3493 (2013)

21. Wang, L., Xu, Q., Zhao, P.: Quantitative estimates on periodic homogenization of nonlinear elliptic operators (2018). arXiv:1807.10865

22. Wang, L., Xu, Q., Zhao, P.: Convergence rates on periodic homogenization of $p$-Laplace type equations (2018). arXiv:1812.04837

23. Zeidler, E., Boron, L.F.: Nonlinear Functional Analysis and Its Applications, Nonlinear Monotone Operators. Springer, New York (1990)

24. Zhao, J.: Homogenization of the boundary value for the Neumann problem. J. Math. Phys. 56, 021508 (2015) 